\title{
Tests for the replication of an association between Egfr and natural variation in Drosophila melanogaster wing morphology Arnar Palsson*1,2, James Dodgson ${ }^{1,3}$, Ian Dworkin ${ }^{1}$ and Greg Gibson ${ }^{1}$
}

Address: ${ }^{1}$ Department of Genetics' North Carolina State University, Raleigh, NC 27695, USA, ${ }^{2}$ Department of Ecology and Evolution, University of Chicago, Chicago, IL 60637, USA and '3The Department of Biochemistry, University of Sussex, Brighton, BN1 9QG, UK

Email: Arnar Palsson* - apalsson@uchicago.edu; James Dodgson - j.dodgson@sussex.ac.uk; Ian Dworkin - i_dworkin@ncsu.edu; Greg Gibson - ggibson@unity.ncsu.edu

* Corresponding author

Published: 15 August 2005

BMC Genetics 2005, 6:44 doi:10.1/86/147|-2156-6-44
Received: 17 March 2005

Accepted: 15 August 2005

This article is available from: http://www.biomedcentral.com/I47/-2/56/6/44

(C) 2005 Palsson et al; licensee BioMed Central Ltd.

This is an Open Access article distributed under the terms of the Creative Commons Attribution License (http://creativecommons.org/licenses/by/2.0), which permits unrestricted use, distribution, and reproduction in any medium, provided the original work is properly cited.

\begin{abstract}
Background: Quantitative differences between individuals stem from a combination of genetic and environmental factors, with the heritable variation being shaped by evolutionary forces. Drosophila wing shape has emerged as an attractive system for genetic dissection of multidimensional traits. We utilize several experimental genetic methods to validation of the contribution of several polymorphisms in the Epidermal growth factor receptor (Egfr) gene to wing shape and size, that were previously mapped in populations of Drosophila melanogaster from North Carolina (NC) and California (CA). This re-evaluation utilized different genetic testcrosses to generate heterozygous individuals with a variety of genetic backgrounds as well as sampling of new alleles from Kenyan stocks.
\end{abstract}

Results: Only one variant, in the Egfr promoter, had replicable effects in all new experiments. However, expanded genotyping of the initial sample of inbred lines rendered the association nonsignificant in the CA population, while it persisted in the NC sample, suggesting population specific modification of the quantitative trait nucleotide QTN effect.

Conclusion: Dissection of quantitative trait variation to the nucleotide level can identify sites with replicable effects as small as one percent of the segregating genetic variation. However, the testcross approach to validate QTNs is both labor intensive and time-consuming, and is probably less useful than resampling of large independent sets of outbred individuals.

\section{Background}

Elucidation of the specific genetic variants that underlie natural phenotypic variation constitutes a major challenge for evolutionary geneticists. Our understanding of evolution will remain incomplete until the relative proportions of deleterious, (nearly) neutral and adaptive factors are documented, in terms of number of loci, their individual and joint effects as well as mode of expression [1]. Several practical issues complicate this endeavor. First, assessment of the contribution of loci and nucleotide variants can be confounded by chance effects, leading to inflated estimates [2]. Second, precise assessment of the effects of segregating polymorphisms on phenotypes depends critically on accurate mapping of the variants, down to individual quantitative trait nucleotides (QTN). Third, environmental interaction, epistasis and pleiotropy, all add complexity to the architecture of genetic variation $[1,3]$. 
Most common implementations of quantitative trait locus (QTL) mapping have low bias with respect to genomic coverage, but only identify allelic variation between two strains. In model organisms, these approaches allow assessment of marginal and epistatic effects, since the experiments are conducted with a large number of offspring, often in laboratory settings that reduce environmental variance. In practice, QTL are rarely resolved to individual loci or exact causal genetic variants [3-5], although several studies on plants offer exceptions [6,7] (reviewed in [8]). In D. melanogaster, QTL loci have also been dissected with quantitative complementation tests $[9,10]$ and/or by linkage disequilibrium (LD) mapping involving a candidate region or locus. These approaches have the resolution to establish a significant contribution of allelic variation at single genes [9,11-20] and even specific nucleotides [21-23].

Successful implication of allelic and nucleotide variation in candidate genes in the production of phenotypic variation is aided by low amounts of $\mathrm{LD}$, due to substantial historical recombination, in the fly genome. LD mapping in D. melanogaster can be implemented with varying degrees of control over genetic and environmental variance from wild caught individuals, laboratory reared iso-female lines, inbred strains, chromosome extraction lines and strains with introgressed chromosome regions. It is now clear that the power and resolution of association studies varies among organisms according to the extent of haplotype structure, and that different experimental approaches must be taken to verify associations in each organism. Despite the lesson from LD mapping in humans that extensive repetition, across cohorts and populations, is crucial to verify allelic contributions [24,25], replication of associations in model organisms is almost non-existent. More research into genetic approaches to validation of QTN effects is needed.

Drosophila wing shape has been used extensively as a model for the study of integration of developmental and quantitative genetics $[26,27]$ and for analysis of the evolution of clinal variation in morphology [28-30]. More specifically, wing shape has proven to be an amenable system for studies on developmental modularity and integration [31], developmental stability [32], selection responses [33-35], laboratory adaptation [36] and more recently for the quantitative genetic dissection of patterning $[23,37$ 41]. Wing shape is commonly described by geometric morphometric tools [42] that capture variation in the locations of landmarks at junctions of veins, cross-veins and the wing margin. The veins have a stereotypical configuration in the Sophophoran family of Drosophilids, with only minor differences documented between species [43], but diversity of shape is considerable $[44,45]$. Wing shape is highly polygenic $[26,33,34,46]$ and we proposed that the spacing and length of veins is a major source of this variation [47].

QTL mapping and quantitative complementation tests support the involvement of venation loci, including components of the EGFR/Ras pathway, in naturally occurring wing shape variation $[38,41]$. These observations led us to test association between allelic variation in the Egfr locus and shape, by sequencing $\sim 11 \mathrm{~kb}$ of the locus in 210 inbred lines from two North American localities, NC and CA $[23,48]$. Significant association of six polymorphisms in Egfr with aspects of wing shape and size, either as main effects or by interaction with population or sex, were reported. A follow-up with wild caught flies confirmed one of the associations, suggesting that QTN effects responsible for less than one percent of the variation for a complex trait can be isolated [49].

The aim of the current study was to assess the capacity of a series of controlled cross designs to validate the contribution of Egfr polymorphisms to naturally occurring variation for wing shape and size. Three schemes were employed, two involving crosses among a subset of the NC lines (a round robin in which 71 nearly isogenic lines were each tested in six random crosses to each other; and a backcross of each of 79 of the lines to two of the most phenotypically extreme lines), and a third involving test crosses between an independent set of Kenyan second chromosomes and the Samarkand wild-type and EgfrE1 and blistered ${ }^{1}$ mutant alleles (Figure 1 ). Only one of the six previously reported associations replicated in all datasets, the variant in the Egfr promoter that showed the most significant main effect in the original study and that also replicated in the wild caught flies [49]. However, when we increased the genotyping in the inbred lines, an interesting dichotomy appeared: the association persisted in the North Carolinian sample but vanished in the Californian population. These results argue for the need of large samples, direct contrast of genetic designs, and most importantly increased replication across populations to fully explore the utility of LD mapping to ascertain nucleotide differences affecting continuous variation of evolutionary importance. They also have implications for the fundamental question of whether quantitative genetic variants have variable effects in different populations [50,51].

\section{Results}

\section{Similarity of shape variation between datasets}

Comparison of genotype-phenotype associations between datasets requires that the phenotypic measurements be comparable. We have adopted principal component (PC) descriptors of shape, and although these are modified subtly by inclusion of more wing data [23] overall the shape metrics extracted from each dataset individually are remarkably similar as depicted for consensus 
Round robin

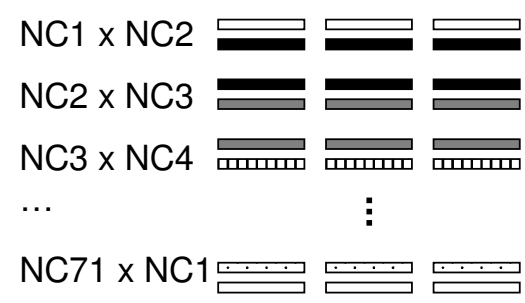

Backcross

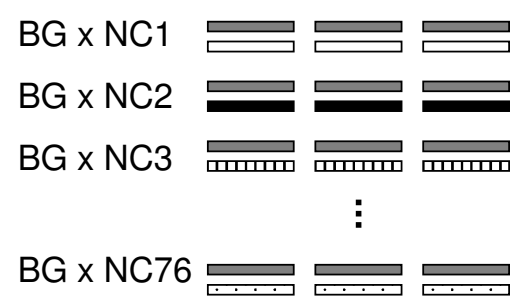

BG: NC025 or NC144
Kenyan testcross

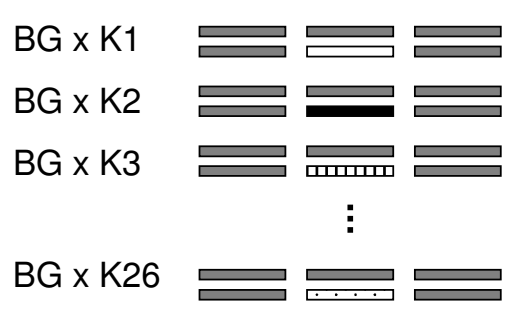

BG: Sam, $b s^{1}$ or $E 1$

\section{Figure I}

Schema of the three experimental crosses. (A) In the round robin (RR), each of $7 \mathrm{I}$ inbred lines from NC was crossed to six other lines to produce heterozygous offspring. Six loops of the type shown were used. (B) In the Backcross (BC), each of 76 $\mathrm{NC}$ lines were crossed to two phenotypically divergent backgrounds, $\mathrm{NC025}$ and NCI44, again resulting in heterozygous offspring. (C) Each of 26 Kenyan second chromosomes extracted into the Samarkand background were crossed to regular Samarkand or Samarkand lines carrying blistered' or EgfrEllipse mutations.

configurations of standardized principal component deviations in Figure 2B-I. This is true both for major (for example C1) and minor (W7) principal components, suggesting that shape variation in North American and African populations of $D$. melanogaster wings reduces to few shared dimensions (see also reference [52]). Furthermore, the eigenvalue decomposition for principal components derived from the individual experiments is qualitatively similar, as shown in Figure 3A. The only exception is the Backcross dataset, where the first PC's for the central region and the whole wing have unusually extreme values. This commonality of the axes of shape variation justified re-extraction of PC's for all datasets jointly, and these joint values were used for all subsequent tests of association. Note that the use of "jointly" or "separately" derived PC's has negligible effect on the test statistics for genetic terms and estimated effects (Table 1 and Additional table 1).

\section{Absence of support for effects of Egfr on wing size}

In order to re-evaluate our previously published associations between wing size and Egfr polymorphisms, recrossing of inbred lines used earlier and testcrosses of additional African chromosomes was carried out. Neither of the two variants affecting size of the wing (C31656T and $\mathrm{T} 40722 \mathrm{C}$ ) in the initial study gave a significant association in any of the three new datasets (Table 1: RR, round robin; $\mathrm{BC}$, backcross; KI, Kenyan introgressions). In the initial study, polymorphism C31656T had the strongest association, a Genotype by Sex interaction $(p=$
0.000002 ) that also exhibited a possible three way interaction of Population, Sex and Genotype $(p=0.001)$. As the three-way interaction was primarily caused by larger difference in the CA than the NC sample [23], the lack of signal in the crossed NC lines is not surprising. Similarly, while T40722C had previously opposite effects on size depending on population, its contribution in the NC population was neither replicated in the $\mathrm{BC}$ and $\mathrm{RR}$ recrossing experiments nor in the Kenyan sample. These results indicate that the previously reported association of Egfr with wing size was likely a false positive even though it was significant after adjustment for the number of multiple comparisons experiment-wide.

\section{Replicable effects of one Egfr variant on wing shape}

The two crossing schemes and the Kenyan introgressions were used to re-evaluate the contribution of four Egfr variants to aspects of wing shape. Only one polymorphism T30200C, was significant and had consistent effects in all of these experiments. This variant resides in the second alternate promoter in a putative GAGA factor binding site, and contributes to the first principal component of the central region of the wing (C1: Table 1 and Figure $2 \mathrm{~B}-\mathrm{E})$. One other polymorphism, C30505A in the same promoter, was also significant in all experiments, but had opposite effects on shape metric W7 in the Kenyan sample compared to the Inbred, $\mathrm{BC}$ and $\mathrm{RR}$ experiments. The inconsistency of the effects casts serious doubt on this association. 


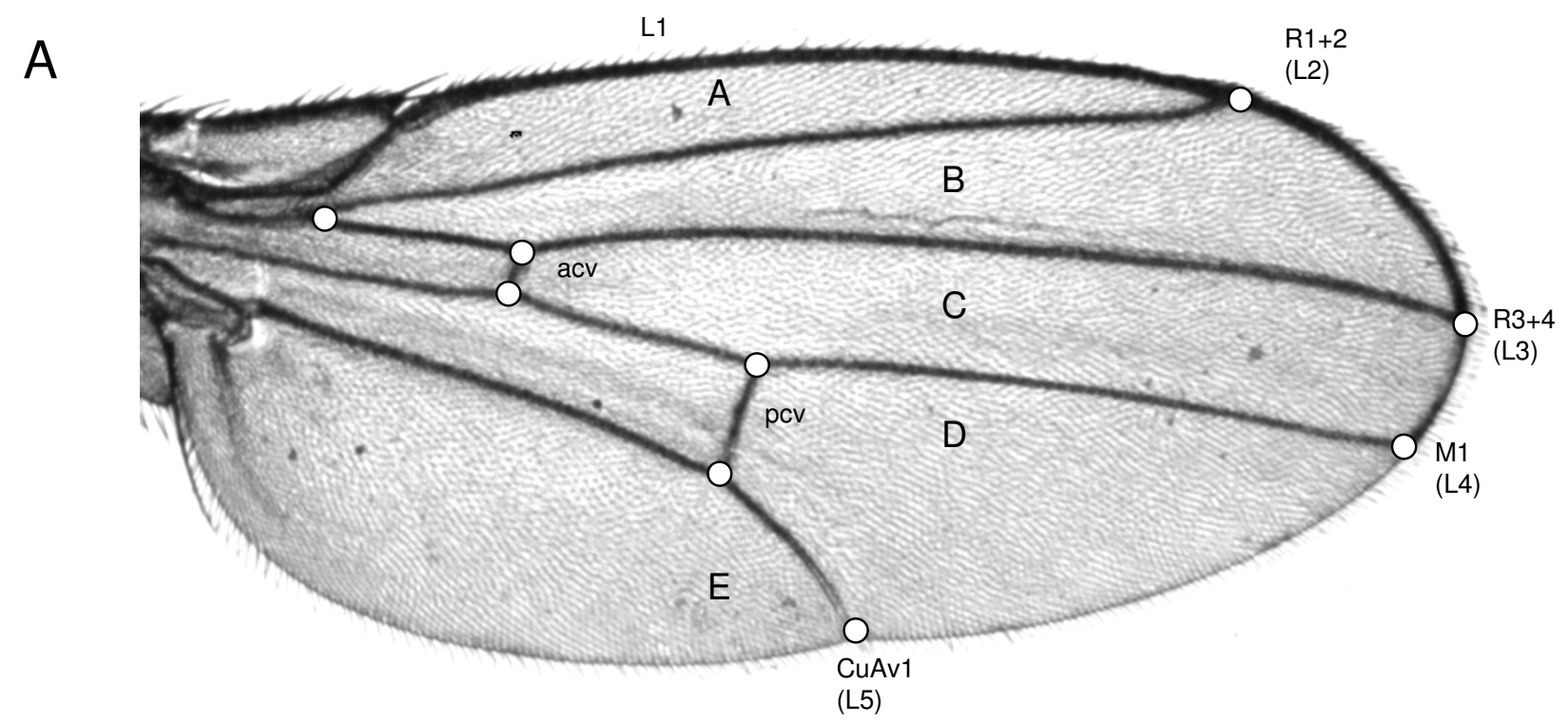

$\mathrm{B}$

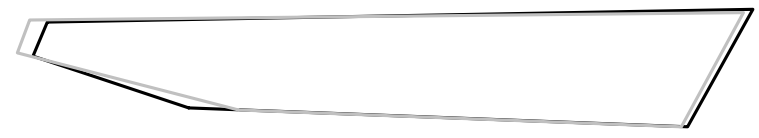

C

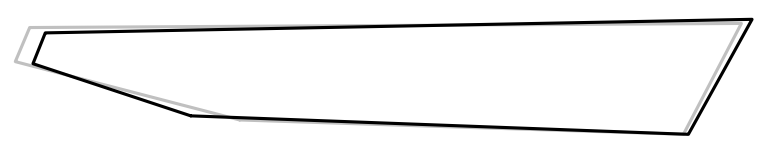

D
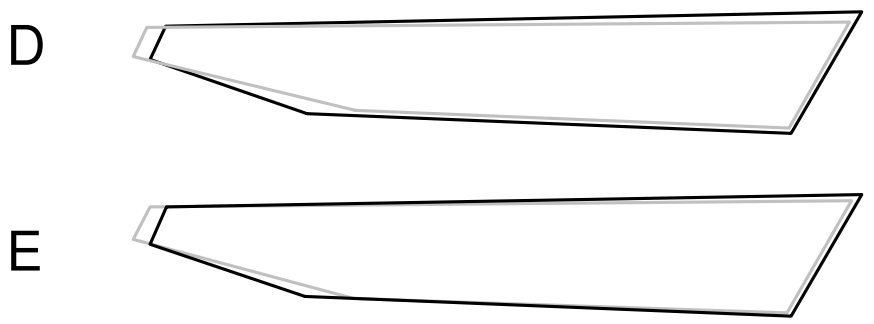

$\mathrm{F}$

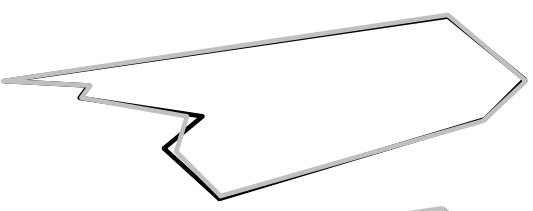

G

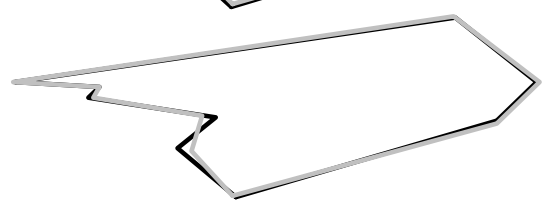

$\mathrm{H}$
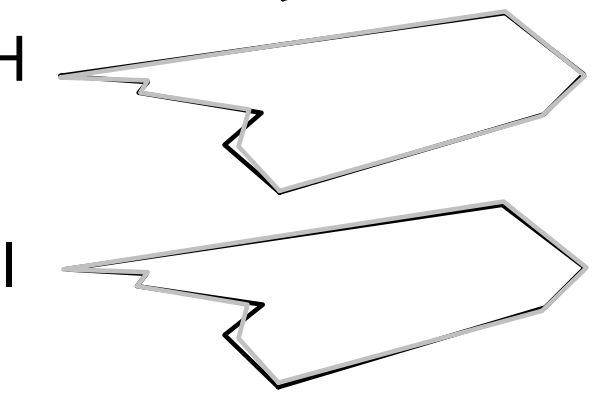

\section{Figure 2}

Shape of the D. melanogaster wing was captured from 9 landmarks $(\mathbf{A})$ and analyzed with TPS software [68]. Veins are labeled both by the Comstock and Needham [69] nomenclature and using developmental genetic terminology (LI-L5). Individual inter-vein regions are designated by the letters A-E. (B - I): Shape differences derived from the four different datasets, namely the inbred $(\mathbf{B}, \mathbf{F})$, backcross $(\mathbf{C}, \mathbf{G})$, round robin $(\mathbf{D}, \mathbf{H})$, and Kenyan $(\mathbf{E}, \mathbf{I})$ panels. B-E shows the first principal component for landmarks of the central region of the wing $(\mathrm{Cl})$. The region is specified by longitudinal veins $\mathrm{L} 3$ and $\mathrm{L} 4$ and the placement of the cross veins and the margin. F-I show the shape differences for the seventh principal component for the whole wing data (W7). The whole wing and the central portion are not drawn to scale. Dark lines represent negative values, and gray positive. For whole wing PC 7 (W7) the extremes are at $+/-0.01$ units and for central region PC I (CI) the values are $+/-0.2$. 

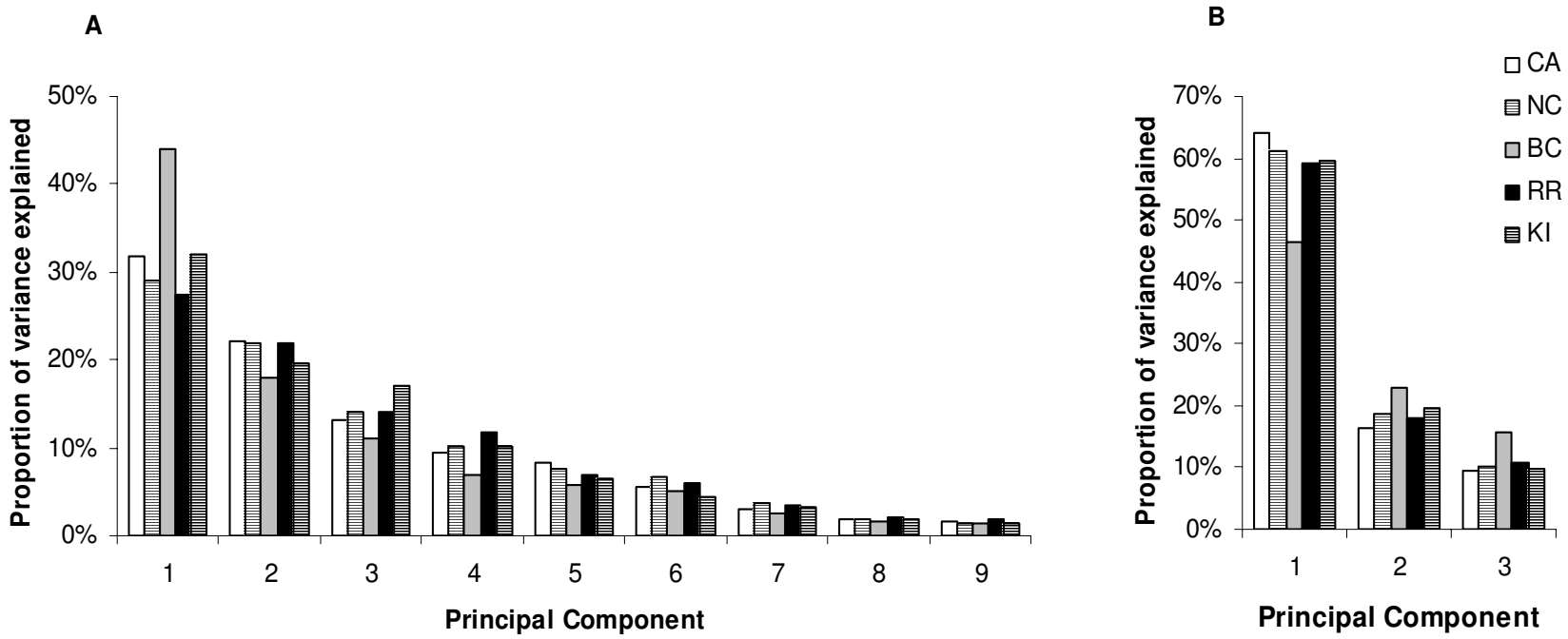

Figure 3

Eigenvalue distribution for the PC's, derived from each of the five datasets individually. (A) Values for the 9 PC's capturing variation in the whole wing, and (B) for the 3 PC's capturing variation for the five landmarks that define the central region of the wing. The decomposition of eigenvalues is comparable for all datasets, with the exception of the Backcross (BC) dataset which deviates qualitatively for the first component in both panels. To illustrate the consistency of shape capture, the principal components for the NC and CA populations were extracted individually for this Figure. All other analyses were conducted on PC's estimated for the CA and NC populations jointly.

Table I: Retesting the effects of Egfr SNP's on wing shape and size

\begin{tabular}{|c|c|c|c|c|c|c|c|c|c|c|c|}
\hline \multirow[b]{2}{*}{ SNP } & \multirow[b]{2}{*}{ Term $^{b}$} & \multirow[b]{2}{*}{ Trait } & \multirow[b]{2}{*}{ Typec } & \multicolumn{4}{|c|}{ Separately derived a } & \multicolumn{4}{|c|}{ Jointly derived a } \\
\hline & & & & INB & $\mathrm{RR}$ & $\mathrm{BC}$ & $\mathrm{KI}$ & INB & $\mathrm{RR}$ & $\mathrm{BC}$ & $\mathrm{KI}$ \\
\hline C3I656T & Gtyp $\times$ Sex & Area & $N$ & $* * * *$ & . & . & . & $* * * *$ & . & . & . \\
\hline C30200Td & Gtyp & $\mathrm{Cl}$ & $N$ & $* * * *$ & $* *$ & $* * * *$ & $* * *$ & $* * * *$ & $* *$ & $* * * *$ & $* * *$ \\
\hline T3I634C & Gtyp $\times$ Sex $\times$ Pop & $\mathrm{C} 2$ & $N$ & $* * * *$ & . & . & . & $* * * *$ & . & . & . \\
\hline T39389C & Gtyp & DI & $\mathrm{S}$ & $* * * *$ & . & . & . & $* * * *$ & . & . & . \\
\hline T40722C & Gtyp $\times$ Pop & Area & $\mathrm{S}$ & $* * * *$ & . & . & na & $* * * *$ & . & . & na \\
\hline C30505A & Gtyp & W7 & $N$ & $* * *$ & $* * *$ & $*$ & $* *$ & $* * *$ & $* * *$ & $* *$ & $*$ \\
\hline
\end{tabular}

a. As described in the Materials and Methods, PC's were calculated for the datasets individually (separately) or for all the data concatenated (jointly).

b. Term denotes the genetic term most significant in the original study. The same term is reported for the repeats, except for the RR where we could only test genotype effects and sites T31634C and T40722C where the genotype terms are reported as population terms are not available. c. Type indicates the nature of the polymorphism, $\mathrm{N}$ : non-coding and S: synonymous.

d. The significance of the $\mathrm{T} 30200 \mathrm{C}$ to $\mathrm{Cl}$ association is here reported for the data from Palsson and Gibson 2004. After re-genotyping the p-values reduce to 0.062 and $0.06 \mathrm{I}$ for the separately and jointly derived data respectively, when analyzed over the NC and CA populations. Note however that the results for RR and BC are for the re-genotyped data. Significance of terms: "." non-significant, "**: $0.05>p>0.01$, "***":p>0.001, "****": $p>$ $0.000 \mathrm{I}$, "*****":p > 0.0000I. P-values are not adjusted to correct for the seventeen new independent tests conducted.

Neither of two other previously reported putative associations [23], the sex and population dependent contribution of site $\mathrm{T} 31634 \mathrm{C}$ to the width of the central region (C2) nor the contribution of T39389C to the posterior region (D1) were supported by the new data. The lack of association of T39389C prompted us to re-examine epistatic effects which included this particular site and associated with variance in the posterior region (D1) of the 
wing [23]. The three site Egfr haplotype (G6065T, T39389C, and T40110C) and also each of the two site haplotypes had given highly significant association in the original panel of inbred lines. Due to smaller sample size in our recrossing datasets, testing of this pattern could only be conducted with the $\mathrm{BC}$ dataset, but the previous epistatic interactions were not confirmed (data not shown). In summary, only one of the Egfr polymorphisms previously implicated to impact wing shape was corroborated by the new data.

\section{Breakdown of the T30200C association in the Californian population}

Previously, due to incomplete genotyping around exon 2, the contribution of T30200C to the central region of the wing was only evaluated with $79 \mathrm{NC}$ and 43 CA lines [23]. Analyses by population found highly significant association in the North Carolinian sample $(p=0.00002)$ but only marginal association in the west coast sample ( $p=$ 0.04) (see Additional Table 1). In order to obtain a better estimate of the magnitude of the effect of T30200C on cross-vein placement, and to investigate the apparent difference in effect between populations, extra genotyping was conducted. The sampling of this polymorphism was increased by re-genotyping the surviving lines from the two populations. Repeating the analysis of variance with $121 \mathrm{NC}$ lines reduced the significance of the association of the T30200C polymorphism $(p=0.002)$. More dramatically, the addition of 30 more alleles to the CA lines $(\mathrm{N}=$ 76) rendered the originally marginal association non-significant $(p=0.9)$ (Additional Table 1 ). Inspection of estimated genotypic effects demonstrates this clearly (Figure 4 and Additional Table 2), as the homozygous classes have nearly identical values for the CA population. Evaluation of the effect of this site in the full dataset without population as a term in the model also renders the association non-significant $(p>0.05)$.

\section{Magnitude of the Egfr allelic contribution}

Estimates of the genotypic effects of T30200C on wing shape are comparable across all of the datasets. There was a slight reduction in observed contribution after the extra genotyping (Additional Table 2), and the estimated difference between homozygote classes was smaller in the RR data than in the NC lines, with the CC and TC heterozygotes being indistinguishable. This suggestion of dominance is opposite that observed in a large sample of outbred flies [49] in which heterozygotes resembled TT homozygotes (dominance was non-zero in this study), but it should be noted that CC homozygotes are very infrequent in the current study. In the $\mathrm{BC}$ experiments, only TT and TC genotypes were available but the magnitude of the difference between genotypic classes was nearly identical in both backcrosses (to NC025 and NC144) and in the RR experiment (Figure 4 and Addi- tional Table 2). The general differences were again of the same magnitude and direction in the testcrosses involving the Kenyan chromosomes, and they scaled additively with the genetic background (Samarkand, E1 or $b s^{1}$ carrying chromosomes).

\section{Experimental designs and potential sites with weak effects} In order to compare the gene-wide patterns of association for each design, the association statistic for the Genotype effect of each site along the Egfr locus is plotted for the three experiments in Figure 5. In each plot, higher significance is toward the top, with thresholds drawn at $p=0.05$ and $p=0.0001$ as before [23]. The analysis focuses on the effects on trait $\mathrm{C} 1$, on basis of the assumption that the T30200C association implicates this shape metric as being affected by variation in Egfr.

The first general result is that the small sample of Kenyan introgressions provides more highly significant sites than the total NC sample (with the exception of T30200C there are no significant associations in common between these two populations). Similarly the RR design yielded more significant test statistics (three sites in the first exon) then the $\mathrm{BC}$ or inbred panels and had 55 sites exceeding the test-wise significance threshold of $p=0.05$. The observed jaggedness of the association profiles likely reflects stochastic fluctuations in the p-values in experiments with relatively small sample size. One interpretation of the data is that the inbred and backcross designs provide better dampening of this stochastic fluctuation then do studies with round robin crossed inbred lines.

The second result is that, in both the RR and BC experiments, the shape of the association profile tracks quite closely with that of the corresponding profile for the set of nearly isogenic lines used to set up the testcrosses. This was not anticipated, since NC025 and NC144 lines have very different wing shapes and each contribute $25 \%$ of the genetic variation in the $\mathrm{BC}$, while the $\mathrm{RR}$ combines the genetic variation of the 71 inbred lines in equal proportions. Evidently genetic correlations between the different testcrosses are sufficient to produce similar association profiles, whether or not these accurately report QTN effects.

Finally, in order to test whether other sites in Egfr affect the cross-vein placement we performed a combined Mixed model ANOVA on the three NC datasets (NC, RR and $\mathrm{BC}$ ). Eleven independent polymorphisms summarized in Table 2 were observed to be significant at the experiment-wide significance level of $p<0.0001$, including site T30200C. Most of these sites are not significant in the CA and Kenyan datasets, but the direction of the genotypic effects generally correspond with the NC panels (only 2/14 are non-concordant, one tailed Fisher exact 


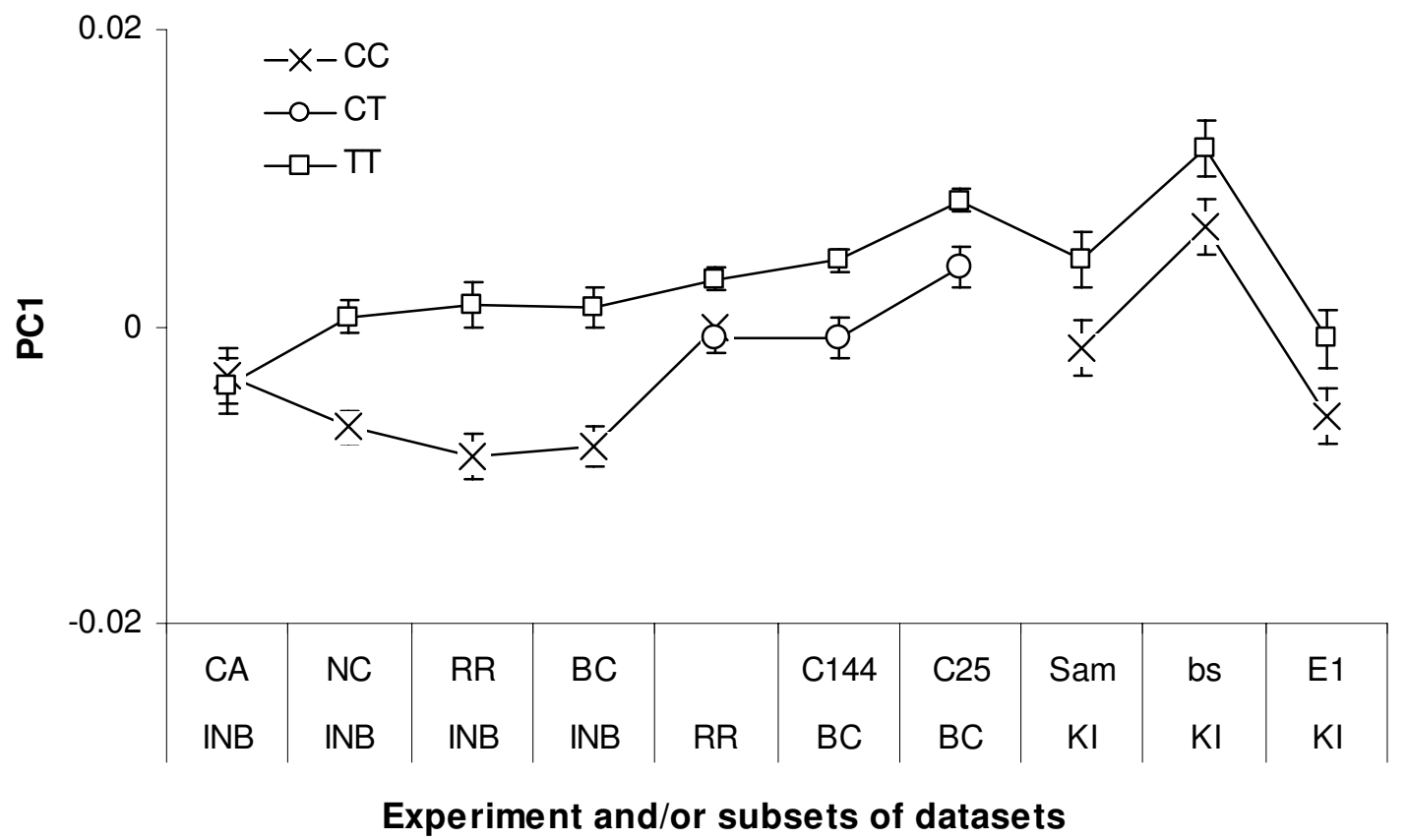

\section{Figure 4}

Effects of the T30200C polymorphism on $\mathrm{Cl}$ in females across experiments and genetic configurations (designated on the $\mathrm{X}$ axis). INB refers to the inbred populations CA and NC, while INB_RR and INB_BC denote the subsets of inbred lines corresponding to the lines used for the recrossing experiments. Likewise, $\mathrm{Cl} 44$ and $\mathrm{C} 25$ indicate the estimated effects of the site in backcrosses to line $\mathrm{NCI} 44$ and $\mathrm{NC025}$ respectively. The last three points show the effects estimates for the three test crosses involving the Kenyan introgressions $(\mathrm{KI})$, namely wildtype Samarkand chromosomes, the blistered' mutant, and the EgfrEllipse allele. Each point represents the least square estimate plus or minus one standard error unit for the indicated homozygous genotypes. See Additional Table 2 for corresponding ANOVA's and values for tests with the older genotype data. The PC's were extracted from all datasets jointly to ensure that the axis of variation and units are comparable.

test yields $p=0.052$ ). Only one of these new candidate variants, C6085G in the less conserved of the two alternate first $\mathrm{N}$-terminal exon, alters the protein, while the remaining are non-coding or silent. Interestingly, one of these silent polymorphisms is C40620T, which also associates with cryptic variation for eye-roughness in inbred lines and wild flies [21]. Note however, if the Egfr variants are tested against other principal component measures of wing shape, similar number of sites emerge at the level of $p<0.0001$ (data not shown) suggesting the caveat that this approach may be inherently noisy.

\section{Discussion}

Previously, fine mapping of the association between polymorphisms in the candidate locus Egfr and wing shape and size in D. melanogaster in 210 inbred lines from two North American populations [23] implicated six Egfr variants or linked polymorphisms as causal variants. In this study we aimed to re-evaluate their involvement through further genetic analysis by generating heterozygous lines derived from crosses of a subset of the original lines and by test crosses with a small sample of African chromosomes. Only one of the retested variants was significant in all datasets and gave consistent effects: the T30200C polymorphism that affects a principal component capturing variation in relative distance between the two cross-veins. 

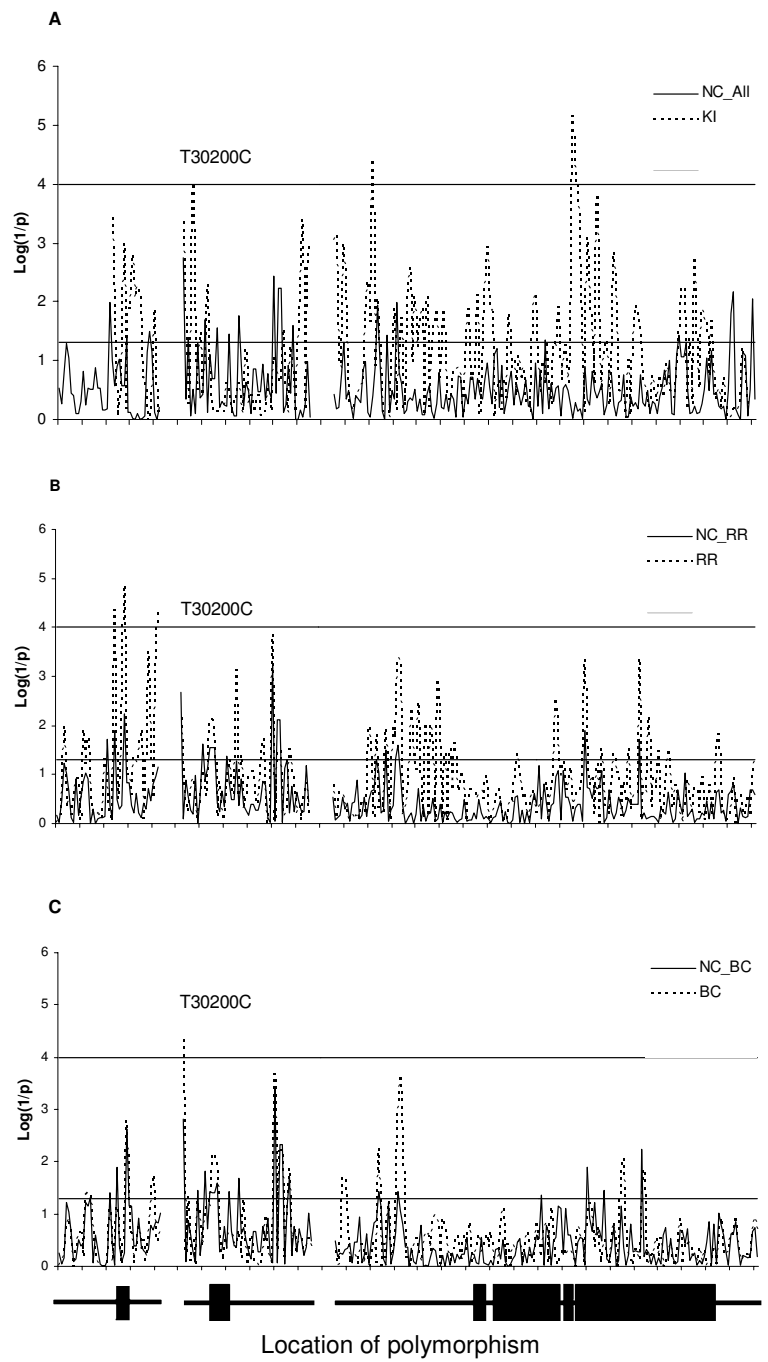

\section{Figure 5}

Association plots for tests of association between Egfr and shape parameter $\mathrm{Cl}$. Each plot shows the negative logarithm of the $p$-value for the test statistic at each polymorphic SNP from 5' to 3 along the Egfr locus. (A) Association profile from the whole $\mathrm{NC}$ inbred panel $(\mathrm{N}=12 \mathrm{I}$, solid line) and the Kenyan chromosomes $(\mathrm{N}=26$, broken line). (B) The association profile for the RR experiment (outcrossed, $\mathrm{N}=7 \mathrm{I}$, broken line) and the subset of $\mathrm{NC}$ lines (in inbred condition, $\mathrm{N}=7 \mathrm{I}$, solid line) used for the outcrossing scheme. (C) Similarly the profiles for the BC panel (backcrossed to NC025 and NCI44, N = 79, broken line) and the corresponding $N C$ set $(N=79$, solid line). The $X$ scale is broken to indicate gaps between contigs with the gene structure represented below (exons as boxes in three contigs of non-coding sequence). Site T30200C (indicated) is located at the 5 ' most end of contig 2. Lines corresponding to single site significance levels $\alpha=0.05$ (negative log $p$ : I.30) and more conservative gene wide $\alpha=0.000$ I (negative log $p: 4.0$ ). Trait values for all lines and crosses come from data that were processed jointly in TPSrelw [68]. 
Table 2: Significance of Egfr polymorphisms on central region shape in NC, CA and Kenyan samples

\begin{tabular}{|c|c|c|c|c|c|c|c|c|}
\hline \multirow[b]{2}{*}{ Site } & \multirow[b]{2}{*}{ Significance in NC } & \multirow[b]{2}{*}{ Location $^{a}$} & \multirow[b]{2}{*}{ Type } & \multicolumn{2}{|c|}{ Effect and significance ${ }^{c}$} & \multicolumn{3}{|c|}{ Frequency of rare allele } \\
\hline & & & & CA & $\mathrm{KI}$ & NC & CA & $\mathrm{K}$ \\
\hline C6085G & $7.98 \times 10^{7}$ & EI & $\mathrm{R}$ & $(\mathrm{OK}) \mathrm{ns}$ & $(\operatorname{Rev})^{n s}$ & $15 / 125$ & $13 / 75$ & $4 / 27$ \\
\hline T30200C & $6.11 \times 10^{10}$ & P2 & $\mathrm{N}$ & $(\operatorname{Rev})^{n s}$ & $(\mathrm{OK}) * * *$ & $26 / 121$ & $21 / 76$ & $8 / 17$ \\
\hline $\mathrm{A} 3 \mathrm{I} 442 \mathrm{~T}$ & $5.49 \times 10^{8}$ & 12 & $\mathrm{~N}$ & $(\mathrm{OK})^{\mathrm{ns}}$ & $(\mathrm{OK})^{\mathrm{ns}}$ & $13 / 92$ & $3 / 24$ & $2 / 20$ \\
\hline A36644T & $9.60 \times 10^{7}$ & 12 & $\mathrm{~N}$ & $(\operatorname{Rev})^{n s}$ & $(\mathrm{OK}) *$ & $26 / 116$ & $3 / 20$ & $9 / 24$ \\
\hline A3676IC & $5.17 \times 10^{6}$ & 12 & $\mathrm{~N}$ & na & na & $35 / 84$ & na & na \\
\hline Del37192d & $3.44 \times 10^{5}$ & 12 & $\mathrm{~N}$ & $(\mathrm{OK})^{\mathrm{ns}}$ & $(\mathrm{OK})^{\mathrm{ns}}$ & $8 / 106$ & $10 / 77$ & $7 / 24$ \\
\hline A37282G & $9.95 \times 10^{6}$ & 12 & $\mathrm{~N}$ & nd & nd & $13 / 106$ & $\mathrm{I} / 77$ & $0 / 23$ \\
\hline T39160C & $2.10 \times 10^{5}$ & E4 & $S$ & $(\mathrm{OK}) \mathrm{ns}$ & (OK) \# & $41 / 128$ & $26 / 76$ & $16 / 35$ \\
\hline $\ln 39534^{d}$ & $8.81 \times 10^{5}$ & 15 & $\mathrm{~N}$ & $(O K)^{n s}$ & $(O K)^{n s}$ & $27 / 110$ & $6 / 27$ & $3 / 33$ \\
\hline C40620Te & $7.37 \times 10^{7}$ & E6 & $S$ & $(\mathrm{OK}) \mathrm{ns}$ & (OK) \# & $46 / 123$ & $19 / 79$ & $7 / 36$ \\
\hline G42242A & $8.88 \times 10^{5}$ & 3' UTR & $N$ & nd & nd & $5 / 105$ & $1 / 12$ & $1 / 25$ \\
\hline
\end{tabular}

a. Location within the Egfr locus, where P2 refers to the second promoter and the other indicators to the respective introns (I), exons (E) and the 3'UTR.

b. Type indicates the nature of the polymorphism; R: replacement, N: non-coding and S: synonymous.

c. Effects of the polymorphisms in the same direction as in NC are indicated by "OK" and those in the reverse direction are designated by "Rev", with the significance of the genotypic term indicated. "ns": not significant, "\#:" $0.1>p>0.05$. "*": $p>0.01$, "**": $p>0.001$, "****": $p>0.000 \mathrm{I}$. P-values are not adjusted to correct for the number of tests conducted. "na": genotypes not available, "nd": not computed because of allele rarity.

d. Del37I 92 is a one base pair deletion and In39534 a four base insert (AACC repeated).

e. Site C40620T is the same as site 8697 described by Dworkin et al. [2I].

However, even the estimated absolute magnitude of this effect is dependent on the survey population and crossing scheme. These results highlight the difficulties in validating weak quantitative effects through experimental genetic approaches and suggest that resampling of outbred populations may be the more conclusive approach to dissection of QTL to the nucleotide level.

\section{The T30200C association persists}

There are at least three possible explanations for the observed restriction of statistical support for the association of T30200C with wing shape to just two of the three populations sampled. The first is that the observed associations in NC and Kenyan samples are false positives, namely that T30200C or linked variants in Egfr do not contribute to shape of the central region of the wing. This seems unlikely, since significant association was also observed in a large sample of outbred NC flies [49] and the association was also replicated in both of the testcross experiments described here.

Two alternative explanations are consistent with the statistical significance being indicative of a true contribution of Egfr polymorphisms to wing shape in NC. One is that the effect of T30200C is masked by genetic variation that is unique to the CA population. Another possibility is that T30200C is not the real causative variant, but exhibits high LD with the causative site in the NC and Kenyan populations but weak LD in the CA population. Since LD in the Egfr decays to background levels over several hundred bases and no differences were observed between NC and CA in their patterns of LD or allele frequencies, while both North American populations diverge considerably from the Kenyan sample [48], this latter explanation is also unlikely. T30200C does not differ in frequency between NC and CA (Fisher's exact test, $p=0.88$ ), but it does lie adjacent to a $23 \mathrm{~kb}$ intron that has not been sequenced in the population sample and could conceivably harbor the true causative variant. However, we favor the hypothesis that one or more modifier loci that differentiate the two North American populations mask the expression of variation due to the Egfr in the CA sample.

Two developmental genetic arguments also lend support to the hypothesis that the $\mathrm{T} 30200 \mathrm{C}$ variant is the causal site. First, our prediction that this site affects a GAGA factor binding element in the Egfr promoter, is supported by genetic interaction between the two loci $[53,54]$. Second, the association between Egfr and cross vein placement is in accord with developmental genetic evidence. Specifically, flies heterozygous for different Egfr alleles lack the majority of the $\mathrm{L} 4$ vein and the entire proximal cross-vein $[53,55,56]$. Recall that shape changes corresponding to principle component $\mathrm{C} 1$ for the central region of the wing (Figure 2B-E) represent variation in the distance between the cross-veins, both of which connect with vein $L 4$. 


\section{Detection of natural alleles with subtle effects}

Quantitative traits in D. melanogaster are now being dissected with QTL mapping, quantitative complementation tests and by testing specific allelic variants by LD mapping. While several studies have found significant association between markers in candidate gene regions and continuous phenotypes [9,11-20] direct re-evaluations of these relationships remain rare. Mackay and Langley [18] found that large insertions around the achaete-scute locus influence bristle number, and this inference was corroborated in a second sample [16]. Geiger-Thornsberry and Mackay [57] confirmed the involvement of two previously identified Delta polymorphisms [15] on bristle number when the same flies were reared under different environmental conditions. Also, we found that three tightly linked silent Egfr polymorphisms affect cryptic variation in eye roughness in inbred lines, and then confirmed the finding in an independent sample of wild caught flies [21]. These studies corroborate the involvement of allelic variation in specific genes with quantitative traits. On the other hand, MacDonald and Long [58] failed to confirm the involvement of a large indel in the 5' region of hairy on bristle number that was previously observed [20]. Moreover, even though both Lai et al. [12] and Lyman et al. [17] implicated scabrous in variation for bristle number, these two studies differed in which markers were typed and by criteria for evaluation of significance (Lai et al. [12] reported an excess of associations with $p$-value below 0.05 while Lyman et al. [17] found three individual significant sites after permutation testing). Finally Genissel et al. [59] asked if the reported Delta bristle association [16] was caused by common replacement polymorphisms in the gene but were not able to identify the hypothesized causal variant.

In summary, several studies have aimed to validate the contribution of allelic to phenotypic variation, but interpretation is complicated by numerous differences between the studies, including: which population is sampled, the genetic designs, the types of genetic markers employed, and control over environmental variation. Additionally, while negative or only weakly suggestive results are sometimes reported [58-61], bias towards publication of positive results may prevent honest evaluation of the nature of the genetic basis of quantitative traits. In theory, once particular polymorphisms have been associated with an evolutionarily important trait, experimental genetic approaches can be used to confirm the functional differences between alleles [62-66]. However, due to technical complexity such methods have yet to be deployed to systematically gauge the effect of segregating variation in Drosophila. In the case of the Egfr, the proposed regulatory regions are too extensive to evaluate the dynamic contribution of allelic variants to vein and intervein determination, so extensive replication is the only viable approach to dissection of QTN effects.

\section{Mapping resolution and experimental designs}

Successful fine mapping of QTL depends on multiple factors such as the magnitude of effect, pattern of LD in the region, available genetic resources, appropriateness of the selection of candidate genes/regions/molecular markers, and the dependence of expression of genetic variation on the experimental settings. The experiments reported here were designed to evaluate the potential for defined crosses to further dissect the role of QTN in subtle quantitative variation, but no obvious recommendations (apart from the need for deep sampling) are forthcoming since the different approaches only produce broadly comparable results.

The round robin and backcross approaches were designed to evaluate the degree to which effects observed in inbred lines are also seen in mixed genetic backgrounds. If the effects of the SNP are additive and there is no epistasis, then they should be just as strong in the testcrosses as in the nearly isogenic lines, with the caveat that there are three genotypes at each SNP to compare instead of just two. The BC design differs in two distinct ways from the RR design, namely the reduced genetic variation (two genomes contribute $50 \%$ of the alleles) and the capacity to detect epistatic effects. This latter could occur by interaction between the QTN and other loci, either due to decanalization as these other loci perturb the phenotype away from the population mean, or simply because QTN effects may generally be so modified by the background that they are only observed in certain backgrounds. The similarity of the estimated genotypic mean differences over the two BC backgrounds and the close tracking of means in the KI experiment (Figure 4), suggests that the reduced genotypic variance is responsible for higher significance of the T30200C association in the BC cross. While this argues for the additivity of the genotypic effects in this case, it is not clear that similar effects will be observed for other traits or loci.

While the ten new highly significant sites in the combined model may be false negatives in the initial lines, more data would be required to confirm that they are true positives. These results indicate that recrossing and deeper population sampling has at best low power to detect novel candidate sites with subtle effects on the phenotype. Consequently, the testcrosses do not obviously outperform the inbred line analysis or bring us any closer to resolving true positive QTN from false positives. Even with a relatively large experiment such as this, the amount of labor and time spent on setting up several hundred crosses and phenotyping several thousand wings does not overcome sampling biases. Even if our analyses suggest 
that other sites in Egfr may affect cross-vein placement, a considerably larger sample than explored here would be required to validate these sites. The testcross results strongly suggest that we can eliminate highly significant results from the first experiment as false positives, but can not conclusively resolve the question of whether the Egfr QTL resolves to a single or several QTN.

\section{Conclusion}

The Egfr contribution to shape variation in D. melanogaster wings reported in Palsson and Gibson [23], and replicated here and in Dworkin et al. [49], represent the best validated example of allelic contribution to continuous morphological variation in flies. While we can not assert that the polymorphism implicated is the causative variant, the evidence and literature cited provide hypotheses testable with experimental genetics. The practical lesson from the observation that five of the six retested Egfr variants failed to validate in testcrosses is that stochastic factors have a substantial impact on analysis of the genetic basis of continuous phenotypes in studies involving fewer than 200 inbred lines. Apparent conditional polymorphisms may be especially sensitive to these effects of chance, and all unreplicated association studies in Drosophila should be considered with this caveat in mind. We suggest that measurement of a very large number of offspring is essential for replication and validation in association studies, and that these are better sampled in outbred wild individuals than in laboratory lines. The declining cost of genotyping will facilitate this transition to large scale mapping of quantitative traits to single nucleotides in ecological settings.

\section{Methods}

\section{Stocks and crossing schemes}

Three separate experiments were conducted to re-evaluate the contribution of Egfr on wing shape (Figure 1). Two involved recrossing, by round-robin (RR) and modified backcross (BC) designs, (71 and 76 NC lines respectively, with 70 being shared). The RR crossing scheme is a partial diallele cross, with the 73 lines being crossed three times as sire and three times as dam. The mating scheme was derived by permutation. In the $\mathrm{BC}$ design, males from 76 NC lines were crossed independently with females from two strains NC025 and NC144. These inbred lines have extreme PC1 values for the anterior and posterior regions of the wing. The third experiment (KI) involved an independent set of Kenyan alleles from Ron Woodruff [48]. Second chromosomes were substituted into the Samarkand background by a 4 generation crossing scheme, utilizing stocks kindly provided by Trudy MacKay. Similarly an Egfr allele, Ellipse (E1), and a blistered allele $\left(b s^{1}\right)$ were substituted into Sam. The wild-type chromosomes were tested over these two mutations and the wild-type Samarkand second chromosomes in three replicate crosses arranged in random blocks. All crosses involved three males crossed to three females, and where conducted in two (RR and BC) or three replicates (KI).

\section{Fly rearing and scoring of wings}

Flies were reared at $25^{\circ} \mathrm{C}$ in standard cornmeal medium with a constant light/dark cycle. Density was controlled by placing two virgin females and two males in a vial and discarding parents on the $2^{\text {nd }}$ or $4^{\text {th }}$ day depending on visual assessment of egg density. The right wing of eight to ten randomly selected individuals per sex (except only RR females) from each vial was scored. In the Kenyan introgression experiment the visible marker $C y$ on the balancer chromosome distinguished genotypes of lines inviable as $2^{\text {nd }}$ chromosome homozygotes. Handling of specimens and data processing was identical to previous experiments [47]. In short, wings were dissected at the hinge and arranged on glass slides and held in place with a cover slip. Within 48 hours, wings were digitally photographed at $4 \times$ magnification with a Spot camera, mounted on a Nikon microscope. Images were processed with Adobe Photoshop version 5, and landmarks captured in Scion Image (freeware available [67]). The nine landmarks at the junctions of veins and wing margin are depicted in Figure 2A. One author, JD, digitized the back-cross and $\sim 65 \%$ of the round-robin while the remaining specimens (35\% of RR, Inbred and Kenyan) were scored by AP. No significant "investigator" effects were found in an analysis of 1000 RR wings scored by both authors (not shown).

\section{Extracting common axes of shape variation}

Shape variation was summarized with the TPSrelw software version 1.39 (freeware available [68]) by calculating relative warps for a set of landmarks, for the whole wing or individual regions (Figure 2A). The procedure involves "partial Procrustes" superimposition, by iterated rotation and alignment of specimens, rescaling to unit size, prior to extraction of the relative warps. The relative warps are essentially principal components (PC's), and will be referred to as such henceforth.

\section{Egfr genotype matrix}

Genotypes used for the association tests were derived from our earlier sequence data [48]. The BC and RR recrossing was not designed to test particular polymorphisms, and therefore generated heterozygotes and sometimes both homozygotes at particular nucleotide positions. For instance in the $\mathrm{BC}$ design, of the six sites retested, T31634C and C30505A were not typed in NC144. Furthermore, of the remaining four polymorphisms, the lines differed only at T40722C. Note this does not mean that their Egfr haplotypes are highly similar, as 167 out the 232 common Egfr sites genotyped in both lines differ, with several recombination events evident. F1 lines that were missing a genotype of one parent where 
omitted from the analysis for that particular genotype. In the Kenyan sample, only the variant T40722C was not tested, as it was only available in one Kenyan line. The Egfr alleles were not sequenced in the three tester chromosomes, leading to tests on haploid data.

\section{Re-genotyping of T30200C}

The T30200C polymorphism in the non-coding region upstream of alternative exon one [48] was re-genotyped in the NC and CA lines in 2004. The previous sample was incomplete due to high level of PCR failure that we attributed to repetitive elements in the region [48]. Therefore an alternative strategy for genotyping was deployed, utilizing the observation that this polymorphism affects a Restriction Length Fragment Polymorphism (RFLP) for the DraIII restriction endonuclease. As before, a single male from each line was genotyped [48]. For PCR, the following new primers were utilized as described in [49]: 5'GTGGCTCGTAATGTGAAACT-3' and 5'-GCGTTACTGGTGGGATGAATCAAG-3'. Of the 210 original lines characterized in 2001-2002, 198 were still surviving in 2004 and were regenotyped. Three discrepancies were found, all in the NC panel (NC065, NC075, NC116). In the case of NC065 heterozygosity for the 3'end of the locus was noted in the original study and it is consequently quite possible that two alleles were segregating when the line was initially genotyped. Contamination of either DNA samples or stocks maintained over this period are also formal possibilities, particularly for the other two lines. These three lines were dropped from the re-analyses.

\section{Analysis of phenotypic variation}

All statistical analysis used SAS version 8.2 (SAS Institute, Cary, NC). The estimation of line effects and extraction of line means was implemented with the LSMEANS option in Proc GLM. The model for the RR dataset was:

$\mathrm{Y}=\mu+$ Line $+\operatorname{Rep}($ Line $)+\varepsilon$

where Line represents each of the $\mathrm{F} 1$ lines generated by the round-robin crosses, and Rep the replicate vial. For the Back-cross and the Kenyan introgression, a more complicated model was used, accounting for the effects of Cross (to NC025 and NC144 or to Sam, E1 and bs), Sex or Line.

$\mathrm{Y}=\mu+$ Cross + Sex $+\mathrm{C} \times \mathrm{S}+$ Line $+\mathrm{S} \times \mathrm{L}+\mathrm{C} \times \mathrm{L}+\mathrm{C} \times \mathrm{S} \times$ $\mathrm{L}+\operatorname{Rep}(\mathrm{C} \times \mathrm{L})+\mathrm{S} \times \mathrm{R}(\mathrm{C} \times \mathrm{L})+\varepsilon$

In both models terms including Line and Rep are considered random. We also performed the analysis without Rep as a term, with the same results.

\section{Tests of quantitative nucleotide effects}

The main aim of these experiments was to re-evaluate the six sites which gave significant signals for wing size and shape in [23]. The RR experiment focused on females from a single population (NC) and a simple model was implemented in Proc Mixed:

$\mathrm{Y}=\mu+$ Gtyp $+\operatorname{Rep}($ Gtyp $)+\varepsilon$

Gtyp is the fixed effect of Genotype, and Rep is a random term, again the replicate vials. For the back-cross and the Kenyan test cross, the model accounted for the contribution of sex and cross:

$\mathrm{Y}=\mu+\mathrm{Gtyp}+\mathrm{Sex}+$ Cross $+\mathrm{G} \times \mathrm{S}+\mathrm{G} \times \mathrm{C}+\mathrm{G} \times \mathrm{S} \times \mathrm{C}+$ Line $(\mathrm{G} \times \mathrm{C})+\varepsilon$

The mean effects of polymorphisms were estimated by the LSMEANS option. Reduced models, by crosses, and extended, by including replicates were also studied and were in accord.

In order to gauge the effects of additional sites in Egfr on the $\mathrm{C} 1$ we utilized a related model, substituting the Cross term with a fixed experiment (Exp) term to demarcate the $\mathrm{NC}, \mathrm{BC}$ and RR datasets, and restricting the analysis to females as the RR panel had no males. The sire and dam are random effects nested within the fixed effects:

$\mathrm{Y}=\mu+\mathrm{Gtyp}+\operatorname{Exp}+\mathrm{G} \times \mathrm{E}+\operatorname{dam} \times \operatorname{sire}(\mathrm{G} \times \mathrm{C})+\operatorname{Rep}(\mathrm{dam}$ $\times$ sire $\times \mathrm{G} \times \mathrm{C})+\varepsilon$

Sites with probability of genotype term below 0.0001 , where then investigated for consistency in genotypic effects and their significance in the CA and KI dataset.

\section{Authors' contributions}

AP, GG and JD designed experiments. JD crossed and scored RR and BC experiments, AP crossed and scored KI, Inbreds and parts of RR dataset. AP conducted statistical analysis. ID regenotyped the T30200C variant. AP, ID and GG wrote the manuscript and all authors approved the final version.

\section{Additional material}

\section{Additional table 1}

ANOVA tables for site T30200C and wing shape. The file shows the results of Analysis of Variance for the T30200C variant in the Egfr promoter and the first principle component for the shape of the central region of the wing.

Click here for file

[http://www.biomedcentral.com/content/supplementary/1471-

2156-6-44-S1.doc] 


\section{Additional Table 2}

Genotypic effects of T30200C on the first PC of the central region of the wing. This table illustrates the genotypic effects (and standard errors) for the T30200C association to wing shape of the central region.

Click here for file

[http://www.biomedcentral.com/content/supplementary/14712156-6-44-S2.doc]

\section{Acknowledgements}

Trudy MacKay kindly provided the Samarkand stocks for the introgression and advice on analysis. Thanks to Marcos Antezana, Lisa Goering, Todd Martin and Jean-Claude Walser for comments on the manuscript.

\section{References}

I. Barton NH, Keightley PD: Understanding quantitative genetic variation. Nat Rev Genet 2002, 3: I I-2I.

2. Beavis WD: The power and deceit of QTL experiments: lessons from comparative QTL studies. Edited by: Washington DC: American Seed Trade Association. 49th Annual Corn and Sorghum Research Conference; 1994:pp 252-268.

3. Mackay TFC: The genetic architecture of quantitative traits. Annual Review of Genetics 200I, 35:303-339.

4. Bolivar VJ, Cook MN, Flaherty L: Mapping of quantitative trait loci with knockout/congenic strains. Genome Research 200I, I I(9): I549-1552.

5. Moore KJ, Nagle DL: Complex trait analysis in the mouse: The strengths, the limitations and the promise yet to come. Annu Rev Genet 2000, 34:653-686.

6. Frary A, Nesbitt TC, Grandillo S, Knaap E, Cong B, Liu J, Meller J, Elber R, Alpert KB, Tanksley SD: fw2.2: a quantitative trait locus key to the evolution of tomato fruit size. Science 2000, 289(5476):85-88.

7. Wilson LM, Whitt SR, Ibanez AM, Rocheford TR, Goodman MM, Buckler 4th ES: Dissection of maize kernel composition and starch production by candidate gene association. Plant Cell 2004, I6(I0):2719-2733.

8. Remington DL, Ungerer MC, Purugganan MD: Map-based cloning of quantitative trait loci: progress and prospects. Genetical Research 200I, 78(3):213-218.

9. De Luca M, Roshina NV, Geiger-Thornsberry GL, Lyman RF, Pasyukova EG, Mackay TFC: Dopa decarboxylase (Ddc) affects variation in Drosophila longevity. Nature Genetics 2003 34(4):429-433.

10. Pasyukova EG, Vieira C, Mackay TF: Deficiency mapping of quantitative trait loci affecting longevity in drosophila melanogaster. Genetics 2000, I 56(3): | | 29-1 | 46.

II. Game AY, Oakeshott JG: Associations between restriction site polymorphism and enzyme activity variation for esterase 6 in Drosophila melanogaster. Genetics 1990, 126(4): I02 I-1031.

12. Lai C, Lyman RF, Long AD, Langley CH, MacKay TFC: Naturally occurring variation in bristle number and DNA polymorphisms at the scabrous locus of Drosophila melanogaster. Science 1994, 266(266): 1697-1702

13. Laurie CC, Bridgeham JT, Choudhary M: Association between DNA sequence variation and variation in expression of the Adh gene in natural populations of Drosophila melanogaster. Genetics 1991, I 29:489-499.

14. Lazzaro BP, Sceurman BK, Clark AG: Genetic basis of natural variation in D. melanogaster antibacterial immunity. Science 2004, 303(5665): 1873-1876.

15. Long AD, Lyman RF, Langley CH, Mackay TFC: Two sites in the Delta gene region contribute to naturally occurring variation in bristle number in Drosophila melanogaster. Genetics 1998, I49(2):999-1017.

16. Long AD, Lyman RF, Morgan AH, Langley $\mathrm{CH}$, Mackay TFC: Both naturally occurring insertions of transposable elements and intermediate frequency polymorphisms at the achaetescute complex are associated with variation in bristle number in Drosophila melanogaster. Genetics 2000, I54(3): $1255-1269$

17. Lyman RF, Lai CQ, Mackay TFC: Linkage disequilibrium mapping of molecular polymorphisms at the scabrous locus associated with naturally occurring variation in bristle number in Drosophila melanogaster. Genetical Research 1999 , 74(3):303-3II.

18. Mackay TFC, Langley $\mathrm{CH}$ : Molecular and phenotypic variation in the achaete-scute region of Drosophila melanogaster. Nature 1990, 348:64-66.

19. Odgers WA, Healy MJ, Oakeshott JG: Nucleotide polymorphism in the 5 ' promoter region of esterase 6 in Drosophila melanogaster and its relationship to enzyme activity variation. Genetics 1995, I4I(I):215-222.

20. Robin $C$, Lyman RF, Long $A D$, Langley $C H$, Mackay TF: hairy: a quantitative trait locus for Drosophila sensory bristle number. Genetics 2002, 162:155-164.

21. Dworkin I, Palsson A, Birdsall K, Gibson G: Evidence that Egfr contributes to cryptic genetic variation for photoreceptor determination in natural populations of Drosophila melanogaster. Current Biology 2003, I 3(2I): |888-1893.

22. Inomata N, Goto H, Itoh M, Isono $K$ : A Single-Amino-Acid Change of the Gustatory Receptor Gene, Gr5a, Has a Major Effect on Trehalose Sensitivity in a Natural Population of Drosophila melanogaster. Genetics 2004, 167(4): 1749- 1758.

23. Palsson A, Gibson G: Association between nucleotide variation in Egfr and wing shape in Drosophila melanogaster. Genetics 2004, 167(3): 1187-1198.

24. Colhoun HM, McKeigue PM, Smith GD: Problems of reporting genetic associations with complex outcomes. Lancet 2003, 361:865-872.

25. loannidis JPA, Ntzani EE, Trikalinos TA, Contopoulos-loannidis DG: Replication validity of genetic association studies. Nature Genetics 200I, 29:306-309.

26. Cowley DE, Atchley WR: Quantitative genetics of Drosophila melanogaster. II. Heritabilities and genetic correlations between sexes for head and thorax traits. Genetics 1988, I I 9:42 I-433 [http://www.genetics.org].

27. Whitlock MC, Fowler $K$ : The changes in genetic and environmental variance with inbreeding in Drosophila melanogaster. Genetics 1999, I 52(I):345-353.

28. Calboli FCF, Gilchrist GW, Partridge L: Different cell size and cell number contribution in two newly established and one ancient body size cline of Drosophila subobscura. Evolution 2003, 57(3):566-573.

29. Gilchrist S, Partridge L: A comparison of the genetic basis of wing size divergence in three parallel body size clines of Drosophila melanogaster. Genetics 1999, I 53(4): 1775- 1787.

30. Huey RB, Gilchrist GW, Carlson ML, Berrigan D, Serra L: Rapid evolution of a geographic cline in size in an introduced fly. Science 2000, 287(545I):308-309.

31. Klingenberg CP, Zaklan SD: Morphological intergration between development compartments in the Drosophila wing. Evolution Int J Org Evolution 2000, 54(4): | 273-I285.

32. Klingenberg CP, Mclntyre GS, Zaklan SD: Left-right asymmetry of fly wings and the evolution of body axes. Proc Biol Sci 1998, 265( (1402): 1255-1259.

33. Weber KE: Selection on wing allometry in Drosophila melanogaster. Genetics 1990, I 26(4):975-989.

34. Weber KE: Increased selection response in larger populations. I. Selection for wing-tip height in Drosophila melanogaster at three population sizes. Genetics 1990 I 25(3):579-584.

35. Weber KE: How small are the smallest selectable domains of form? Genetics 1992, I30(2):345-353.

36. Santos M, Iriarte PF, Cespedes W, Balanya J, Fontdevila A, Serra L: Swift laboratory thermal evolution of wing shape (but not size) in Drosophila subobscura and its relationship with chromosomal inversion polymorphism. Journal of Evolutionary Biology 2004, I7(4):84I-558.

37. Mezey JG, Houle D, Nuzhdin SV: Naturally Segregating Quantitative Trait Loci Affecting Wing Shape of Drosophila melanogaster. Genetics 2005, 169(4):2101-21।3.

38. Palsson A, Gibson G: Quantitative developmental genetic analysis reveals that the ancestral dipteran wing vein prepattern 
is conserved in Drosophila melanogaster. Dev Genes Evol 2000, 210(12):617-622.

39. Weber K, Eisman R, Higgins S, Morey L, Patty A, Tausek M, Zeng ZB: An analysis of polygenes affecting wing shape on chromosome 2 in Drosophila melanogaster. Genetics 200I, 159:1045-1057.

40. Weber KE, Eisman R, Morey L, Patty A, Sparks J, Tausek M, Zeng ZB: An analysis of polygenes affecting wing shape on chromosome 3 in Drosophila melanogaster. Genetics 1999, 1 53:773-786.

41. Zimmerman E, Palsson A, Gibson G: Quantitative trait loci affecting components of wing shape in Drosophila melanogaster. Genetics 2000, 155(2):67I-683.

42. Bookstein FL: Morphometric tools for landmark data: geometry and biology. Cambridge, Massachusetts, Cambridge University Press; 1991:435.

43. Stark J, Bonacum J, Remsen J, DeSalle R: The evolution and development of dipteran wing veins: a systematic approach. Annu Rev Entomol 1999, 44:97-129.

44. Galpern P: The use of common principle component analysis in studies of phenotypic evolution: an example from the Drosophilidae. In Zoology Toronto , University of Toronto; 2000:|3|.

45. Houle D, Mezey JG, Galpern P, Carter A: Automated measurement of Drosophila wings. BMC Evolutionary Biology 2003, 3(I):25

46. Weber K, Johnson N, Champlin D, Patty A: Many P-Element Insertions Affect Wing Shape in Drosophila melanogaster. Genetics 2005, 169(3): | 46|-1475.

47. Birdsall K, Zimmerman E, Teeter K, Gibson G: Genetic variation for the positioning of wing veins in Drosophila melanogaster. Evol Dev 2000, 2(I):16-24.

48. Palsson A, Rouse A, Riley-Berger R, Dworkin I, Gibson G: Nucleotide variation in the Egfr locus of Drosophila melanogaster. Genetics 2004, 167(3): I 199-1212.

49. Dworkin I, Palsson A, Gibson G: Replication of an Egfr-Wing Shape Association in a Wild-Caught Cohort of Drosophila melanogaster. Genetics 2005, 169(4):21/5-2I25.

50. Goldstein DB, Hirschhorn JN: In genetic control of disease, does 'race' matter? Nat Genet 2004, 36( ( 2): I 243-1244

5I. loannidis JP, Ntzani EE, Trikalinos TA: 'Racial' differences in genetic effects for complex diseases. Nat Genet 2004, 36(12): $|3| 2-13 \mid 8$.

52. Mezey JG, Houle D: The dimensionality of genetic variation for wing shape in Drosophila melanogaster. Evolution 2005, in press:.

53. Angulo M, Corominas M, Serras F: Activation and repression activities of ash2 in Drosophila wing imaginal discs. Development 2004, I 3 I(20):4943-4953.

54. Bejarano F, Busturia A: Function of the Trithorax-like gene during Drosophila development. Developmental Biology 2004 268(2):327-34I.

55. Diaz-Benjumea FJ, Garcia-Bellido A: Behaviour of cells mutant for an EGF receptor homologue of Drosophila in genetic mosaics. Proc Biol Sci 1990, 242(I 303):36-44.

56. Sturtevant MA, Roark M, Bier E: The Drosophila rhomboid gene mediates the localized formation of wing veins and interacts genetically with components of the EGF-R signaling pathway. Genes Dev 1993, 7(6):961-973.

57. Geiger-Thornsberry GL, MacKay TFC: Association of singlenucleotide polymorphisms at the Delta locus with genotype by environment interaction for sensory bristle number in Drosophila melanogaster. Genetical Research 2002, 79:2II-2I8.

58. Macdonald SJ, Long AD: A potential regulatory polymorphism upstream of hairy is not associated with bristle number variation in wild-caught Drosophila. Genetics 2004, 167(4):2|27-2|3|.

59. Genissel A, Pastinen T, Dowell A, Mackay TF, Long AD: No evidence for an association between common nonsynonymous polymorphisms in delta and bristle number variation in natural and laboratory populations of Drosophila melanogaster. Genetics 2004, 166(I):291-306.

60. Goering LM, Gibson G: Genetic variation for dorsal-ventral patterning of the Drosophila melanogaster eggshell. Evol dev 2005, 7(2):8I-88.
61. Nikoh N, Duty A, Gibson G: Effects of population structure and sex on association between serotonin receptors and Drosophila heart rate. Genetics 2004, I68(4): 1963-1974.

62. Choudhary M, Laurie CC: Use of in vitro mutagenesis to analyze the molecular basis of the difference in Adh expression associated with the allozyme polymorphism in Drosophila melanogaster. Genetics 199I, 129:48I-488.

63. Greenberg AJ, Moran JR, Coyne JA, Wu Cl: Ecological adaptation during incipient speciation revealed by precise gene replacement. Science 2003, 302(565 I): I754-1757.

64. Laurie CC, Stam LF: The Effect of an Intronic Polymorphism on Alcohol Dehydrogenase Expression in Drosophila melanogaster. Genetics 1994, 138:379-385.

65. Odgers WA, Aquadro CF, Coppin CW, Healy MJ, Oakeshott JG: Nucleotide polymorphism in the Est6 promoter, which is widespread in derived populations of Drosophila melanogaster, changes the level of Esterase 6 expressed in the male ejaculatory duct. Genetics 2002, 162(2):785-797.

66. Stam LF, Laurie CC: Molecular dissection of a major gene effect on a quantitative trait: The level of alcohol dehydrogenase expression in Drosophila melanogaster. Genetics 1996, 144(4): I559-1564.

67. Scion Image [http://www.scioncorp.com]

68. Rohlf, F. J. TPS relative warp analysis software Windows [http://life.bio.sunysb.edu/morph/index.html].

69. Comstock JH, Needham JG: The wings of insects. American Naturalist 1898, 32:43-903.
Publish with Biomed Central and every scientist can read your work free of charge

"BioMed Central will be the most significant development for disseminating the results of biomedical research in our lifetime. "

Sir Paul Nurse, Cancer Research UK

Your research papers will be:

- available free of charge to the entire biomedical community

- peer reviewed and published immediately upon acceptance

- cited in PubMed and archived on PubMed Central

- yours - you keep the copyright

Submit your manuscript here:

http://www.biomedcentral.com/info/publishing_adv.asp
BioMedcentral 